\title{
Buffer-gas loading of atoms and molecules into a magnetic trap
}

\section{Citation}

Doyle, John M., Bretislav Friedrich, Jinha Kim, and David Patterson. 1995. Buffer-gas loading of atoms and molecules into a magnetic trap. Physical Review A 52(4): R2515-2518.

\section{Published Version}

http://dx.doi.org/10.1103/PhysRevA.52.R2515

\section{Permanent link}

http://nrs.harvard.edu/urn-3:HUL.InstRepos:2710571

\section{Terms of Use}

This article was downloaded from Harvard University's DASH repository, and is made available under the terms and conditions applicable to Other Posted Material, as set forth at http:// nrs.harvard.edu/urn-3:HUL.InstRepos:dash.current.terms-of-use\#LAA

\section{Share Your Story}

The Harvard community has made this article openly available.

Please share how this access benefits you. Submit a story.

Accessibility 


\title{
Buffer-gas loading of atoms and molecules into a magnetic trap
}

\author{
John M. Doyle, ${ }^{1}$ Bretislav Friedrich, ${ }^{2}$ Jinha Kim, ${ }^{1}$ and David Patterson ${ }^{1}$ \\ ${ }^{1}$ Department of Physics, Lyman Laboratory, Harvard University, Cambridge, Massachusetts 02138 \\ ${ }^{2}$ Department of Chemistry, 12 Oxford Street, Harvard University, Cambridge, Massachusetts 02138
}

(Received 6 June 1995)

\begin{abstract}
We describe a method for loading paramagnetic atoms or molecules into a magnetic trap. A ${ }^{3} \mathrm{He}$ buffer gas is employed to thermalize atoms or molecules to a temperature of approximately $240 \mathrm{mK}$, lower than the depth of the trap. A model is described that indicates an initial loading density of approximately $10^{13} \mathrm{~cm}^{-3}$. Once loading has taken place the buffer gas is removed by cryopumping. Evaporative cooling can then be applied to further lower the temperature and increase the density of the trapped sample.
\end{abstract}

PACS number(s): 32.80.Pj, 33.80.Ps

Magnetic trapping and evaporative cooling are now established tools in atomic physics. The first magnetic trapping of neutral atoms took place in 1985 when laser cooling of a thermal beam of sodium atoms was used to load an "antiHelmholtz" trap [1]. In the same year, evaporative cooling, a method to further cool trapped atoms, was proposed by Hess $[2,3]$. Soon thereafter, evaporative cooling was demonstrated $[4,5]$ and used to cool a gas of magnetically trapped atomic hydrogen to a temperature of $100 \mu \mathrm{K}$ at the simultaneously attained density of $8 \times 10^{13} \mathrm{~cm}^{-3}$ [6]. Magnetic trapping and evaporative cooling are at the forefront of the search for Bose-Einstein condensation in atomic hydrogen, sodium, and rubidium [7-12]. The development of ultrahigh precision spectroscopy of the $1 S-2 S$ transition in $\mathrm{H}$ also utilizes these techniques [13-15].

The technique of evaporative cooling has found utility for a number of reasons: (1) a large decrease in temperature is achieved while incurring a small decrease in the total number of trapped atoms; (2) the density of the sample can be increased while the sample is cooled; (3) it has general applicability to any atomic species; and (4) effects due to radiation are absent (for example, the single photon recoil limit present in most laser-cooling schemes). In spite of this, evaporative cooling of neutral species has been applied only to atomic hydrogen and some alkali-metal atoms. Only highdensity ensembles of trapped species can be evaporatively cooled, and, so far, this has been achieved only with the alkali-metal atom and hydrogen.

To load a trap to a high density, an efficient cooling mechanism is required. In the case of alkali-metal atoms, laser cooling is used. Unfortunately, laser cooling favors atoms with simple level schemes and accessible excitation wavelengths (in the visible or near-infrared regions). This is particularly problematic for molecules. In the case of atomic hydrogen, interatomic scattering is used in combination with the (unique) low binding energy $(1 \mathrm{~K})$ that $\mathrm{H}$ has to a liquid He surface. Unfortunately, all other species have too high a surface binding energy (with any surface) for this latter technique to be employed; at the low temperatures necessary to load the trap, atoms or molecules would simply stick to the walls.
We describe here a technique for loading into a magnetic trap any paramagnetic atom or molecule with a magnetic dipole moment of at least 1 Bohr magneton $\left(\mu_{B}\right)$ [16]. This is accomplished at low temperature $(\approx 240 \mathrm{mK})$ and at high densities $\left(\approx 10^{13} \mathrm{~cm}^{-3}\right)$. Given these initial loading conditions, it should be possible to evaporatively cool the trapped sample to a temperature of $1 \mu \mathrm{K}$ or below while maintaining or increasing its number density.

The confining potential of a magnetic trap is due to the interaction of the atomic or molecular space-fixed magnetic dipole moment with an inhomogeneous static magnetic field (in what follows the paramagnetic species will be exemplified by a molecule in general and by molecular oxygen in particular). A static magnetic trap can be formed by creating a magnetic-field minimum in free space (by, for example, using an anti-Helmholtz coil configuration). Molecular states whose energy increases with increasing field strength can be trapped. Molecules in states whose energy decreases with field strength are repelled from the trap and discarded.

We propose using a pulsed low-temperature radiofrequency discharge as a source of molecules. The discharge serves to desorb molecules from a frozen solid that coats the discharge walls. Although it operates at around $0.3 \mathrm{~K}$, it can dissociate molecules into atoms. This allows for the possibility of bringing molecules or atoms out of a precursor. The low-temperature discharge has been used to produce atomic hydrogen from frozen molecular hydrogen: atomic hydrogen fluxes of $10^{12} \mathrm{~s}^{-1}$ have been achieved at low temperatures [17-19]. The temperature of neutral species leaving the discharge is expected to be $10^{3} \mathrm{~K}$ or less [20].

In order to load a molecule into the conservative field of a magnetic trap it is necessary to remove energy from the molecule while it is in the trap region. The requisite dissipation of translational energy can be achieved via collisions with a cold buffer gas. In order to ensure that the molecules thermalize (and are trapped) before colliding with the wall of the container surrounding the trap (the "cell"), the density of the buffer gas must be high enough to make the mean free path of the molecule smaller than the size of the cell $(5 \mathrm{~cm}$ in the proposed trap design described below). (Room- 
temperature buffer gases have been previously used to load ion traps [21]. This was realizable with ions because the electrostatic interaction allows very deep traps to be created.)

We have developed a model (outlined below) for the thermalization of hot $\mathrm{O}_{2}$ molecules in a cold ${ }^{3} \mathrm{He}$ buffer gas and have found that under our proposed conditions the molecules will thermalize before touching the cell wall. The vapor pressure of ${ }^{3} \mathrm{He}$ at $240 \mathrm{mK}$ provides a number density of $5 \times 10^{15} \mathrm{~cm}^{-3}$ [22]. Calculation of the mean free path of $\mathrm{O}_{2}$ in ${ }^{3} \mathrm{He}$ requires knowledge of the elastic scattering cross section. This has been measured for velocities corresponding to temperatures between $10^{3}$ and $10^{4} \mathrm{~K}$ [23]. Under the assumptions that the cross section remains at its $10^{3}-\mathrm{K}$ value of $150 \AA^{2}$ for temperatures below $10^{3} \mathrm{~K}$ and that the molecules come from the source at $10^{3} \mathrm{~K}$, the $\mathrm{O}_{2}$ molecule will, on average, thermalize after traveling less than $8 \mathrm{~mm}$ (56 collisions). It is expected that, in fact, the scattering cross section will continue to increase below $10^{3}$ $\mathrm{K}$ before eventually leveling off [24], so $8 \mathrm{~mm}$ is an upper bound.

The total number of molecules loaded into the trap is determined by a balance between the flux of molecules from the discharge and the loss of molecules due to readsorption on the cell walls. The loss of molecules over the lip of the trap is given by the rate at which molecules at the edge of the trap collide with the physical walls (assuming that any molecule impinging on the wall is lost). The total loss rate is given by $n v A / 4$, where $n$ is the density at the wall, $v$ is the average velocity of molecules, and $A$ is the area of the surface defined by the trap edge. The density at the edge of the trap is given by $n=n_{0} e^{-\eta}$, where $\eta \equiv\left|\langle\mu\rangle \mathscr{H}_{\max }\right| k T, n_{0}$ is the density at the center of the trap, $\langle\mu\rangle$ is the space-fixed magnetic moment of the molecule, $\mathscr{H}_{\max }$ is the magnetic field strength at the trap edge, $T$ is the translational temperature of the gas, and $k$ is the Boltzmann constant. The total number, $N$, of trapped molecules, is related to $n_{0}$ by $N=n_{0} V$, where $V$ is the "effective volume" of the trap. $V$ is determined by the temperature of the molecules and the geometry of the trapping fields. For one proposed trap design ( $5 \mathrm{~cm}$ diameter and $4 \mathrm{~T}$ deep) the effective volume is calculated to be $V=V_{0} \eta^{-3}$ with $V_{0}=390 \mathrm{~cm}^{3}$. This expression is sufficiently accurate for $\eta>5$ [25]. Based on a flux of $10^{12}$ $\mathrm{s}^{-1}$, the expected molecular oxygen density just after initial loading is calculated to be more than $10^{17} \mathrm{~cm}^{-3}$. As will be shown below, the initial loading density is probably limited to less than this by other loss mechanisms. For species with $|\langle\mu\rangle|=1 \mu_{B}$, the number density is limited to about $n_{0}=6$ $\times 10^{13} \mathrm{~cm}^{-3}$. The total number trapped in the latter case would then be $N=1 \times 10^{13}$ at the initial loading temperature of $240 \mathrm{mK}$.

The density in the trap may be limited (especially for molecules with $|\langle\mu\rangle| \geqslant 2 \mu_{B}$ ) by loss mechanisms such as intermolecular dipolar relaxation, molecule to buffergas dipolar relaxation, molecule to buffer-gas dimerization [26], and collisional reorientation [27-29]. Intermolecular dipolar relaxation occurs when during a collision the electronic magnetic moments of the molecules interact and one of the molecules makes a transition to the untrapped highfield-seeking state. The low-temperature rate constant for atomic hydrogen has been calculated and measured to be
$1 \times 10^{-15} \mathrm{~cm}^{3} \mathrm{~s}^{-1}[18,30-32]$. At low collision velocities the rate for other species (such as molecular oxygen) could be sensitive to the exact shape of the intermolecular potential; this makes it difficult to draw accurate analogies with other systems, such as atomic hydrogen. Assuming that the dipolar relaxation rate is 100 times larger than for atomic hydrogen, then the limit to the density would be $7 \times 10^{13}$ $\mathrm{cm}^{-3}$.

Due to the very small magnetic moment of ${ }^{3} \mathrm{He}$, the rate of dipolar relaxation in collisions with the buffer gas should be small. An estimate based on scaling by the ratio of the magnetic moment of the helium atom to that of the hydrogen atom indicates that the rate is negligible. The actual rate may depend on the buffer-gas-molecule interaction potential and thus it is prudent to assume that it could be much higher. If the relaxation rate were 100 times higher than indicated by our simple scaling, it would just start affecting the calculated initial density of $7 \times 10^{13} \mathrm{~cm}^{-3}$.

Dimerization of the molecules with the helium atoms could also put a limit on the initial density. However, in order to form the dimer, a three-body collision is necessary. A simple estimate of the rate of ternary collisions for molecular oxygen using the hard-sphere cross sections at a loading temperature of $240 \mathrm{mK}$ shows it to be negligible; dimer production sets on at densities $10^{3}$ times higher than those available during the loading process [26]. Based on the results of Refs. [27-29], collisional realignment should also be negligible.

Majorana transitions are also present due to the zero field at the exact center of the trap. We have estimated the lifetime of the trapped sample to be more than $10^{3} \mathrm{~s}$ at the initial loading temperature. Once cooled to low temperatures, the molecules can be easily moved into a trap with a nonzero minimum, eliminating this slow loss.

Once a steady-state number of molecules in the trap is reached, the buffer gas is withdrawn and the molecules are stored within the trap. The buffer gas can be withdrawn simply by lowering the temperature of the cell; cooling to

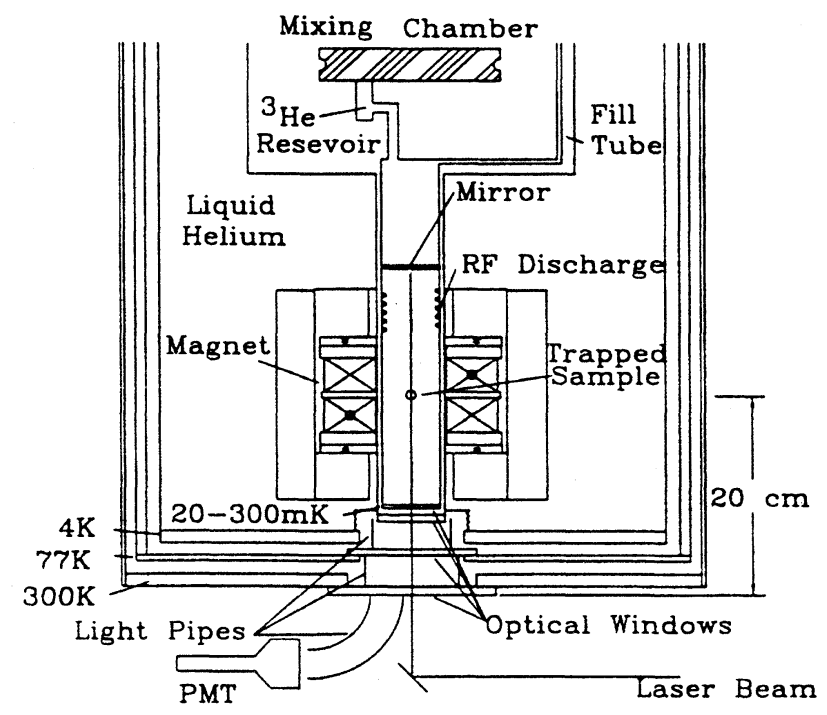

FIG. 1. A possible experimental setup. 
TABLE I. Maximum magnetic dipole moments of selected ground-state atoms arranged by term $[45,46]$. Here $S, L$, and $J$ are spin, orbital, and total electronic angular momentum quantum numbers, respectively, and $g_{J}$ is the Landé factor.

\begin{tabular}{lcc}
\hline \hline State ${ }^{2 S+1} L_{J}$ & $g_{J} J$ & Element \\
\hline${ }^{2} S_{1 / 2}$ & 1.001 & $\mathrm{H}, \mathrm{Li}, \mathrm{Na}, \mathrm{K}, \mathrm{Cu}, \mathrm{Rb}, \mathrm{Ag}, \mathrm{Cs}, \mathrm{Au}, \mathrm{Fr}$ \\
${ }^{4} S_{3 / 2}$ & 3.003 & $\mathrm{~N}, \mathrm{As}, \mathrm{Sb}, \mathrm{Bi}$ \\
${ }^{6} S_{5 / 2}$ & 5.006 & $\mathrm{Mo}, \mathrm{Cr}$ \\
${ }^{7} S_{3}$ & 6.007 & $\mathrm{Eu}$ \\
${ }^{8} S_{7 / 2}$ & 7.007 & $\mathrm{~B}, \mathrm{Al}, \mathrm{Ga}, \mathrm{In}, \mathrm{Tl}$ \\
${ }^{2} P_{1 / 2}$ & 0.333 & $\mathrm{~F}, \mathrm{Cl}, \mathrm{Br}, \mathrm{I}, \mathrm{At}$ \\
${ }^{2} P_{3 / 2}$ & 2.001 & $\mathrm{O}, \mathrm{Se}, \mathrm{Te}, \mathrm{Po}$ \\
${ }^{3} P_{2}$ & 3.002 & $\mathrm{Nb}$ \\
${ }^{6} D_{1 / 2}$ & 1.669 & $\mathrm{Sc}, \mathrm{Y}, \mathrm{La}, \mathrm{Lu}, \mathrm{Ac}$ \\
${ }^{2} D_{3 / 2}$ & 1.199 & $\mathrm{Pt}$ \\
${ }^{3} D_{3}$ & 4.002 & $\mathrm{Gd}$ \\
${ }^{9} D_{2}$ & 5.303 & $\mathrm{Fe}, \mathrm{Os}$ \\
${ }^{5} D_{4}$ & 6.005 & $\mathrm{Ti}, \mathrm{Zr}, \mathrm{Hf}$ \\
${ }^{4} F_{3 / 2}$ & 0.598 & $\mathrm{Tm}$ \\
${ }^{3} F_{2}$ & 1.332 & $\mathrm{Ni}$ \\
${ }^{2} F_{7 / 2}$ & 3.994 & $\mathrm{Co}, \mathrm{Rh}, \mathrm{Ir}$ \\
${ }^{3} F_{4}$ & 5.002 & $\mathrm{Ru}$ \\
${ }^{4} F_{9 / 2}$ & 6.003 & $\mathrm{Ce}$ \\
${ }^{5} F_{5}$ & 7.005 & $\mathrm{Pm}$ \\
${ }^{1} G_{4}$ & 3.782 & $\mathrm{Er}$ \\
${ }^{6} H_{5 / 2}$ & 3.782 & $\mathrm{~Tb}$ \\
${ }^{3} H_{6}$ & 6.983 & $\mathrm{Nd}$ \\
${ }^{6} H_{15 / 2}$ & 9.938 & $\mathrm{Pr}$ \\
${ }^{5} I_{4}$ & 2.413 & $\mathrm{Dy}$ \\
${ }^{4} I_{9 / 2}$ & 3.290 & \\
${ }^{4} I_{15 / 2}$ & 8.964 & ${ }^{5} I_{8}$ \\
\hline \hline
\end{tabular}

$80 \mathrm{mK}$ reduces the vapor pressure below $10^{-15}$ Torr. At this pressure the collision time of the trapped molecules with the background gas will be greater than $10^{7} \mathrm{~s}$, resulting in a negligible loss rate.

Once the buffer gas is removed the trapped molecules will continue colliding with each other-they will thermalize. Under these conditions evaporative cooling can be employed to lower the temperature of the molecules even further. How efficiently this can be done is determined by the ratio of the elastic and dipolar relaxation cross sections [33,34]. Calculations of the ultra-low-temperature dipolar and elastic cross sections are, in general, sensitive to the exact shape of the intermolecular interaction potentials [35] and have not been attempted for a species like molecular oxygen. It is, however, expected (and has been experimentally verified for some alkali atoms) that, in general, the ratio of the elastic to the dipolar cross section should increase with increasing size of the trapped species. Atomic hydrogen (which has a very small elastic cross section) has been evaporatively cooled to $100 \mu \mathrm{K}$ and it should be possible to cool it to $1 \mu \mathrm{K}$ [11]. Atomic sodium and atomic rubidium have been evaporatively cooled to $1 \mu \mathrm{K}$ and $200 n \mathrm{~K}$, respectively [36,7]. Thus, the ultimate temperature reachable for other species (such as molecular oxygen) is likely to be lower than $1 \mu \mathrm{K}$.
A sketch of a possible experimental apparatus is shown in Fig. 1. The trap magnet itself is of anti-Helmholtz configuration and its coils are wound with standard $\mathrm{Nb}$-Ti superconducting wire. The field at the physical wall can be made $4 \mathrm{~T}$ using readily available technology. The magnet is immersed in liquid helium and surrounds a "vacuum can" that in turn surrounds the low-temperature cell. The cell is connected to the mixing chamber of a dilution refrigerator and thus its temperature can be varied between about 20 and $500 \mathrm{mK}$. The vacuum space between the vacuum can and the cell ensures thermal isolation. Optical access (used to detect the trapped sample using laser fluorescence or absorption) is provided by a set of windows.

During the loading phase of the experiment the temperature of the mixing chamber (and therefore of the cell) is raised to $240 \mathrm{mK}$ and the ${ }^{3} \mathrm{He}$ is free to flow over the entire low-temperature volume. Molecules frozen on the cell walls are liberated by the $\mathrm{rf}$ discharge. Once the molecules are loaded, the mixing chamber is cooled and all of the ${ }^{3} \mathrm{He}$ resides in the reservoir. Due to the strong confinement of the trap, the removal of the buffer gas results in an insignificant loss of molecules.

The proposed technique would provide a new tool for doing atomic and molecular spectroscopy and studying molecular structure, especially ultra-high-resolution spectroscopy that requires cold (slow), trapped (long interaction time) samples. For example, it should be possible to trap and cool $\mathrm{YbF}$, a paramagnetic molecule proposed for use in the search for an elementary electric dipole moment (a test of time reversal symmetry) [37,38]. Using cold, trapped YbF would greatly increase the sensitivity of such experiments.

There is also interest in collisional relaxation of molecules at very low energies $[39,40]$. The production of ultracold molecules would allow the study of collisions where the translational energy is smaller than the other relevant energy scales in the system. The creation of low-temperature samples of atoms and molecules may also allow loading of the far-off-resonance trap $[41,42]$.

In the atomic domain, the technique could be applied to a host of different paramagnetic atoms (from anywhere in the Periodic Table). One prominent example is atomic deuterium. So far, serious attempts to trap it have failed [43]. Trapped atomic deuterium, however, could play a very prominent role in high-precision testing of quantum electrodynamics [44].

We have surveyed the Periodic Table and have found that more than half of all ground-state atoms should be trappable by this technique, cf. Table I. Particularly interesting is the fact that a significant number of atoms have maximum magnetic moments much higher than $1 \mu_{B}$. This allows for the possibility of loading a magnetic trap at temperatures around $1 \mathrm{~K}$, accessible by a simple pumped liquid helium system.

In summary, we have described a proposed method for loading paramagnetic atoms and molecules into a magnetic trap. Once loaded into the trap it should be possible to evaporatively cool the sample to $1 \mu \mathrm{K}$ or less. This would allow high-precision spectroscopy to be done on a new host of atoms and molecules. 
[1] A. L. Migdall et al., Phys. Rev. Lett. 54, 2596 (1985).

[2] H. F. Hess, Bull. Am. Phys. Soc. 30, 854 (1985).

[3] H. Hess, Phys. Rev. B 34, 3476 (1986).

[4] N. Masuhara et al., Phys. Rev. Lett. 61, 935 (1988).

[5] H. F. Hess et al., Phys. Rev. Lett. 59, 672 (1987).

[6] J. M. Doyle et al., Phys. Rev. Lett. 67, 603 (1991).

[7] W. Petrich et al., Phys. Rev. Lett. 74, 3352 (1995).

[8] T. J. Greytak, in Bose-Einstein Condensation, edited by A. Griffin, D. W. Snoke, and S. Stringari (Cambridge University Press, Cambridge, 1995), p. 131.

[9] K. B. Davis et al., Phys. Rev. Lett. 74, 5202 (1995).

[10] C. S. Adams, H. J. Lee, M. Kasevich, and S. Chu, Phys. Rev. Lett. 74, 3577 (1995).

[11] I. D. Setija et al., in Atomic Physics 14, Proceedings of the Fourteenth International Conference on Atomic Physics, edited by D. J. Wineland, C. E. Wieman, and S. J. Smith (AIP, New York, 1995), p. 389.

[12] I. Setija, Ph.D. thesis, Universiteit van Amsterdam, 1995 (unpublished).

[13] T. W. Hänsch, in Atomic Physics 14, (Ref. [13]), p. 63.

[14] J. C. Sandberg, Ph.D. thesis, Massachusetts Institute of Technology, 1993 (unpublished).

[15] D. Kleppner, in The Hydrogen Atom, edited by G. F. Bassani, M. Inguscio, and T. W. Hänsch (Springer-Verlag, Berlin, 1989), p. 103.

[16] J. M. Doyle, Bull. Am. Phys. Soc. 39, 1166 (1994).

[17] H. F. Hess et al., Phys. Rev. A 34, R34 (1986).

[18] J. Helffrich, M. Maley, M. Krusius, and J. Wheatley, J. Low Temp. Phys. 66, 277 (1987)

[19] R. van Roijen, J. J. Berkhout, S. Jaakkola, and J. T. M. Walraven, Phys. Rev. Lett. 61, 931 (1988).

[20] V. M. Donnelly, D. Flamm, and G. Collins, J. Vac. Sci. Technol. 21, 817 (1982).

[21] J. N. Louris, J. W. Amy, T. Y. Ridley, and R. G. Cooks, Int. J. Mass Spectrom. Ion Processes 88, 97 (1989), and references contained within.

[22] J. Wilks, The Properties of Liquid and Solid Helium (Clarendon Press, Oxford, 1987).

[23] F. P. E. Luzzatti and F. Vecchiocattivi, Mol. Phys. 34, 1279 (1977).

[24] H. S. W. Massey and C. B. O. Mohr, Proc. R. Soc. (London) A144, 188 (1934).

[25] J. M. Doyle, Ph.D. thesis, Massachusetts Institute of Technology, 1991 (unpublished).

[26] D. R. Miller, in Atomic and Molecular Beam Methods, edited by G. Scoles (Oxford University Press, New York, 1988), p. 40.

[27] M. J. Weida and D. J. Nesbitt, J. Chem. Phys. 100, 6372 (1994).

[28] D. Pullman, B. Friedrich, and D. Herschbach, J. Chem. Phys. 93, 3224 (1990).

[29] A. J. McCassery, J. Chem. Phys. 91, 5451 (1987).

[30] H. T. C. Stoof, J. M. V. A. Koelman, and B. J. Verhaar, Phys. Rev. B 38, 4688 (1988).

[31] A. Lagendijk, I. F. Silvera, and B. J. Verhaar, Phys. Rev. B 33, 626 (1986).

[32] J. M. Doyle et al., J. Opt. Soc. Am. B 6, 2244 (1989).

[33] J. M. Doyle et al., in Physica B, 20th International Conference on Low-temperature Physics, edited by R. J. Donnelly (Elsevier, Amsterdam, 1994), p. 13.

[34] J. Luiten, Ph.D. thesis, Universiteit van Amsterdam, 1993 (unpublished).

[35] J. Weiner, J. Opt. Soc. Am. B 6, 2270 (1989).

[36] K. B. Davis (private communication).

[37] M. G. Kozlov and V. F. Ezhov, Phys. Rev. A 49, 4502 (1994).

[38] E. A. Hinds and K. Sangster, in Time Reversal-The Arthur Rich Memorial Symposium, edited by M. Skalsey, P. H. Bucksbaum, R. S. Conti, and D. W. Gidley, AIP Conf. Proc. No. 270 (AIP, New York, 1993), p. 77.

[39] D. W. Noid, S. K. Gray, and S. A. Rice, J. Chem. Phys. 84, 2649 (1986).

[40] S. K. Gray and S. A. Rice, J. Chem. Phys. 83, 2818 (1985).

[41] J. D. Miller, R. A. Cline, and D. J. Heinzen, Phys. Rev. A 47, R4567 (1993).

[42] B. Friedrich and D. Herschbach, Phys. Rev. Lett. 74, 4623 (1995).

[43] O. J. Luiten and J. M. Doyle, loading of deuterium into the magnetic traps at both University of Amsterdam and MIT was attempted but failed. This was attributed to the high binding energy of deuterium to the liquid helium surface and to the high cross length for surface recombination.

[44] F. Schmidt-Kaler et al., in Atomic Physics 13, Thirteenth International Conference on Atomic Physics, edited by $\mathrm{H}$. Walther, T. W. Hänsch, and B. Neizert (AIP, New York, 1992), p. 31.

[45] C. E. Moore, Atomic Energy Levels, Natl. Bur. Stand. (U.S.) Circ. No. NRDS-NBS35 (U.S. GPO, Washington, D.C., 1971), Vols. 1 and 3.

[46] C. E. Moore, Atomic Energy Levels-The Rare Earth Elements, Natl. Bur. Stand. (U.S.) Circ. No. NSRDS-NBS60 (U.S. GPO, Washington, D.C., 1978). 